\title{
Photodynamic Therapy in a 3D Model of Ovarian Cancer
}

\author{
Shubhankar Nath ${ }^{1, *}$ and Kaitlin Moore ${ }^{2}$
}

\author{
${ }^{1}$ Wellman Center for Photomedicine, Massachusetts General Hospital-Harvard Medical School, Boston, \\ USA; ${ }^{2}$ Bouve College of Health Sciences, Northeastern University, Boston, USA \\ *For correspondence: snath.vet2000@gmail.com
}

\begin{abstract}
[Abstract] Photodynamic therapy (PDT), is a clinically-approved light-based anti-cancer treatment modality in which a photoactivatable photosensitizer is irradiated with an appropriate wavelength of light to generate cytotoxic molecules to kill cancer cells. In this article, we describe an in vitro PDT protocol using a 3-dimensional (3D) model of ovarian cancer that was established on beds of Matrigel. PDT was performed using a liposomal formulation of verteporfin photosensitizer (Visudyne ${ }^{\circledR}$ ). The cancer cells were genetically-labeled with the fluorescent protein mCherry to facilitate the evaluation of the treatment response. This protocol is advantageous because the mCherry fluorescence is an indicator of cell viability, eliminating the need for external dyes, which often exhibit limited penetration and diffusion into 3D organoids. Additionally, Visudyne PDT achieves significant tumor-killing efficacy in a 3D model for ovarian cancer.
\end{abstract}

Keywords: Photodynamic therapy, 3D model, Ovarian cancer, Photosensitizer, Visudyne, Light-based therapies

[Background] Photodynamic therapy (PDT) is an FDA-approved light-based treatment modality that utilizes a photoactivatable molecule, termed a photosensitizer (PS). Upon irradiation with an appropriate wavelength of light, the PS generates reactive molecular species (RMS) within the cytosol. These RMS, often reactive oxygen species, induce photodamage and are toxic to the cell (Celli et al., 2010; Obaid et al., 2016). Certain formulations of PS preferentially accumulate in the malignant tissue and thus PDT often achieves dual selectivity as a result of confined PS accumulation in the tumor and its spatiotemporal control over the light irradiation. PDT is clinically approved for several indications, including esophageal cancer, lung cancer, age-related macular degeneration, and is being investigated for many more cancerous and noncancerous indications (Celli et al., 2010).

Ovarian cancer is a deadly gynecologic malignancy often associated with treatment-resistant metastases and poor outcomes in patients. While surgery and chemotherapy are the current standard of care for ovarian cancer, the efficacy of PDT to manage ovarian cancer is currently being investigated in pre-clinical studies by many research groups (Goff et al., 1996; Rizvi et al., 2010).

Like many other malignancies, ovarian cancer metastases display complex morphological and biochemical characteristics that are lost in traditional monolayer (2D) cell culture. 3D tumor model is an emerging technique for evaluating tumor properties and treatment response in vitro. 3D cultures of tumor cells create a more physiologically relevant tumor microenvironment than traditional 2D cell culture because they retain the biological and structural features of tumors that are found in vivo (Rizvi et al., 
2016). Cells in monolayer experience contact inhibition and a homogeneous oxygen and nutrient environment, while $3 \mathrm{D}$ cultures display heterogeneity within and across spheroids and restore extracellular matrix-related cues that are important in treatment response. Like in vivo tumors, 3D cultured tumors proliferate into heterogeneous clusters with varying levels of oxygen and nutrients, making them useful tools for studying PS uptake and PDT efficacy within oxygen gradients. Increased PDT resistance has been found in 3D tumor models compared with 2D, providing a platform for testing alternative treatment regimens and combination therapies to overcome this resistance. The complex factors that contribute to treatment failure in patients necessitate an in vitro model that mimics the structural, biological, and extracellular cues that play a major role in treatment outcomes (Rizvi et al., 2016).

Here, we describe an in vitro PDT protocol using a 3D model of ovarian cancer. 3D nodules of human ovarian cancer cells ( $\mathrm{NIH}$ : Ovcar5) that stably express a fluorescent reporter were grown on beds of Growth Factor-Reduced Matrige ${ }^{\circledR}$. PDT was performed using FDA-approved PS verteporfin (Visudyne ${ }^{\circledR}$ ). The treatment response was evaluated by imaging of the fluorescent $3 D$ nodules. The protocol described here has been adapted from a previously published article (Rizvi et al., 2019).

\section{Materials and Reagents}

1. Pipette tips (200 and $1,000 \mu \mathrm{l}$, Fisher Scientific, catalog numbers: $02-707-407$ and $02-707-413$, respectively)

2. LASER-safety glasses (Thorlabs, catalog number: LG3)

3. Aspirating pipettes (Celltreat, catalog number: 229265)

4. 5 and $10 \mathrm{ml}$ serological pipettes (Corning, catalog numbers: 357543 and 357551 , respectively)

5. $15 \mathrm{ml}$ centrifuge tubes (Corning, catalog number: 352095)

6. $50 \mathrm{ml}$ centrifuge tubes (Corning, catalog number: 430829)

7. 24-well glass-bottom black-wall plate (Greiner Sensoplates, catalog number: 662892)

8. T75 flask (Corning, catalog number: 353136)

9. Aluminum foil (Fisher Scientific, 01-213-105)

10. mCherry-expressing NIH: OVCAR5 cell line (Rizvi et al., 2019) ("mCherry-Ovcar5" thereafter)

11. Visudyne ${ }^{\circledR}$ (Verteporfin for injection by Bausch \& Lomb)

12. Dulbecco's Phosphate Buffered Saline (DPBS) without $\mathrm{Ca}^{2+} \& \mathrm{Mg}^{2+}$ (Corning, Cellgro, catalog number: 21-031-CV)

13. Heat inactivated fetal bovine serum (FBS) (Gibco, catalog number: 26140079)

14. 100x Penicillin-streptomycin solution (Corning, catalog number: $30001 \mathrm{Cl}$ )

15. Trypsin-EDTA solution (Corning Cellgro, catalog number: $25-052-\mathrm{Cl}$ )

16. Corning Growth factor-reduced (GFR) Matrige ${ }^{\circledR}$ Matrix ("Matrigel” thereafter) (Corning, catalog number: 354230)

17. Matrigel matrix using Cell Recovery Solution (Corning, catalog number: 354253 )

18. RPMI 1640 medium (Corning, Cellgro, catalog number: 10-040-CV) 
19. DMSO (Sigma, catalog number: D2438)

20. RPMI Growth Medium (see Recipes)

21. $4 \%$ Matrigel (see Recipes)

22. Visudyne ${ }^{\circledR}$ working solution (see Recipes)

\section{Equipment}

1. Pipettes (P1000 and P200, Gilson, catalog numbers: F123602 and F123601, respectively)

2. Biosafety cabinet for cell culture (Baker Company, catalog number: SG-200)

3. Pipette-aid (Biotang, catalog number: 710932)

4. Analytical balance (Sartorius, catalog number: ENTRIS2241SUS)

5. Inverted light microscope (VWR, catalog number: 89404-462)

6. 5x air objective (NA 0.16) lens (PerkinElmer, catalog number: HH14000402)

7. Freezer $\left(-20^{\circ} \mathrm{C}\right)$ (Thermo Scientific, model: 20LFEETSA)

8. LASER Source $(690 \mathrm{~nm}$ ) (Intense Ltd., model: Intense series 7400)

9. Laser power meter (Ophir Vega, catalog number: 7Z01560)

10. Laser shutter controller (INLINE-TTL Electronic Shutter)

11. Centrifuge machine (Beckman, model: Allegra 6R)

12. High-Content Imaging Instrument (Operetta CLS, PerkinEImer)

13. Humidified cell incubator (Thermo Scientific, model: Forma ${ }^{T M} 310$ )

14. Cell counter (Beckman Coulter, model: Z1 Particle Counter)

\section{Software}

1. Harmony 4.6 (PerkinElmer)

2. GraphPad Prism 8

3. (Optional) ImageJ or qVista software

\section{Procedure}

Note: Perform all the cell culture-related work inside a sterile biosafety cabinet following standard aseptic technique.

A. Preparation of Matrigel Plate

1. Thaw a vial of Matrigel overnight by submerging the vial in ice in a walk-in cold room.

2. Place a box of sterile pipette tips $(1,000 \mu \mathrm{l})$ and a glass-bottom black-wall 24 -well plate on ice or ice pack for at least 15 min before handling Matrigel.

3. Using a P1000 pipette, gently dispense $250 \mu$ l of Matrigel into each well without introducing any bubbles while keeping the plate on a flat ice pack.

4. Incubate the plate at $37^{\circ} \mathrm{C}$ in a cell culture incubator for 20-30 min to polymerize Matrigel. 
5. Add $500 \mu \mathrm{l} /$ well of RPMI growth medium (see Recipes) containing $4 \%$ Matrigel to avoid further drying of the Matrigel beds.

6. Keep the plate in the cell culture incubator until the mCherry-Ovcar5 cells are ready in Procedure B.

B. Cell seeding and 3D culture

1. Maintain a 2D culture of mCherry-Ovcar5 cells in a T75 flask with RPMI growth medium in a humidified cell culture incubator at $37^{\circ} \mathrm{C}$ with $5 \% \mathrm{CO}_{2}$.

2. Check the confluency of mCherry-Ovcar5 cells using an inverted light microscope. Use a plate that has $50 \%-80 \%$ confluency.

3. Replace the existing culture medium from the flask with $10 \mathrm{ml}$ DPBS and incubate for $10 \mathrm{~min}$.

4. Discard DPBS and add 2-3 $\mathrm{ml}$ of Trypsin-EDTA solution to the flask.

5. Incubate for $3-5 \min$ at $37^{\circ} \mathrm{C}$.

6. Observe the plate under a light microscope to check if the cells come off the surface completely.

7. Quench trypsin by adding $10 \mathrm{ml} \mathrm{RMPI}$ growth medium and resuspend the cells.

8. Transfer the cell suspension into a $15 \mathrm{ml}$ tube.

9. Centrifuge the tube at $450 \times \mathrm{g}$ for $5 \mathrm{~min}$ at room temperature.

10. Discard the supernatant and resuspend the cell pellet with $10 \mathrm{ml}$ RPMI growth medium.

11. Determine the cell concentration using Beckman Coulter Counter by setting the cell size between 8-20 $\mu \mathrm{m}$.

12. Prepare $13 \mathrm{ml}$ cell suspension in RPMl growth medium to achieve a concentration of 10,000 cells $/ 500 \mu \mathrm{l}$.

13. Add $500 \mu \mathrm{l}$ of the cell suspension into each well of the 24 -well plate resulting in the final concentration of 10,000 cells in $1 \mathrm{ml}$ medium with $2 \%$ Matrigel.

14. Incubate the plate for 7 days to allow 3D tumor nodule formation and replace the medium with fresh RPMI growth medium with 2\% Matrigel on Day 4.

C. In vitro Photodynamic therapy

1. On Day 7, observe the plate under an inverted light microscope to ensure the formation of the tumor nodules on the Matrigel beds (see Figure 1A).

2. Prepare $13 \mathrm{ml}$ of Visudyne working solution (see Recipes) by diluting Visudyne stock in RPMI growth medium to achieve $1 \mu$ M PS concentration.

3. Protect the PS solution in the dark and perform the following steps in the dark or low-light environment.

4. Set a very slow aspiration speed and connect a P200 pipette tip (without cotton/filter) to an aspiration pipette and gently aspirate the existing medium from each well without disrupting the 3D nodules.

5. Add $500 \mu \mathrm{l}$ of Visudyne working solution into each well except 'no treatment' and 'light-only' control wells. Add $500 \mu \mathrm{l}$ of RPMI growth medium into each of the control wells. 
6. Incubate for $90 \mathrm{~min}$ at $37^{\circ} \mathrm{C}$ in a cell culture incubator.

7. Replace Visudyne-containing medium with fresh RPMI growth medium.

8. During the PS incubation, prepare the Laser source and controller by adjusting light density (irradiance) to $50 \mathrm{~mW} / \mathrm{cm}^{2}$ using a Laser power meter.

9. To perform PDT, sequentially irradiate each well with $690 \mathrm{~nm}$ light directly administered vertically through the bottom of the plate at different doses of light (for example $0-10 \mathrm{~J} / \mathrm{cm}^{2}$ ) by varying the exposure time using a custom-made programmable TTL shutter.

Note: Do not apply any Laser in the 'no treatment' wells whereas apply the highest dose of Laser into the 'light-only' control wells.

10. Incubate the plate for additional 4 days following the light irradiation.

D. Imaging

1. On Day 11 , image the plate to quantify residual viable tumor by analyzing $m$ Cherry fluorescence (see Figure 1B).

2. Place the plate on Operetta CLS, a high-content imaging instrument with confocal capability while maintaining the temperature at $37^{\circ} \mathrm{C}$ with $5 \% \mathrm{CO}_{2}$.

3. Use the excitation and emission filters of $530-560 \mathrm{~nm}$ and $570-650 \mathrm{~nm}$, respectively to image mCherry-expressing cells.

4. Set image acquisition parameters in Z-stack mode by keeping the visually best fit focal plane in the middle of 10-14 z-planes with $50 \mu \mathrm{m}$ step size.

5. Adjust the LED power and exposure time based on the "No Treatment" control groups so that the camera sensor is not saturated leaving a wide dynamic range for capturing the highest and lowest.

6. Acquire images using a $5 x$ air objective (NA 0.16) lens in a 2-by-2 mosaic-format ( $512 \times 512$ pixels each with $10 \%$ overlap).

\section{Data analysis}

1. Use Harmony 4.6 (PerkinElmer) software that comes with Operetta CLS for image analysis.

2. Stitch the image-mosaics from the same plane first and then generate 2-dimensional "maximum intensity projection" (MIP) to get a global image for each well.

3. Set a threshold for mCherry fluorescence based on the "no treatment control" to determine the "live tumor area."

4. Export the values for residual tumor area as a cvs. file and prepare presentable graphs using other software (such as Microsoft ${ }^{\circledR}$ Excel or GraphPad Prism) (see Figure 1C)

\section{Representative Results}

Representative outcomes from a PDT treatment of 3D ovarian tumor nodules are shown in Figure 1. 


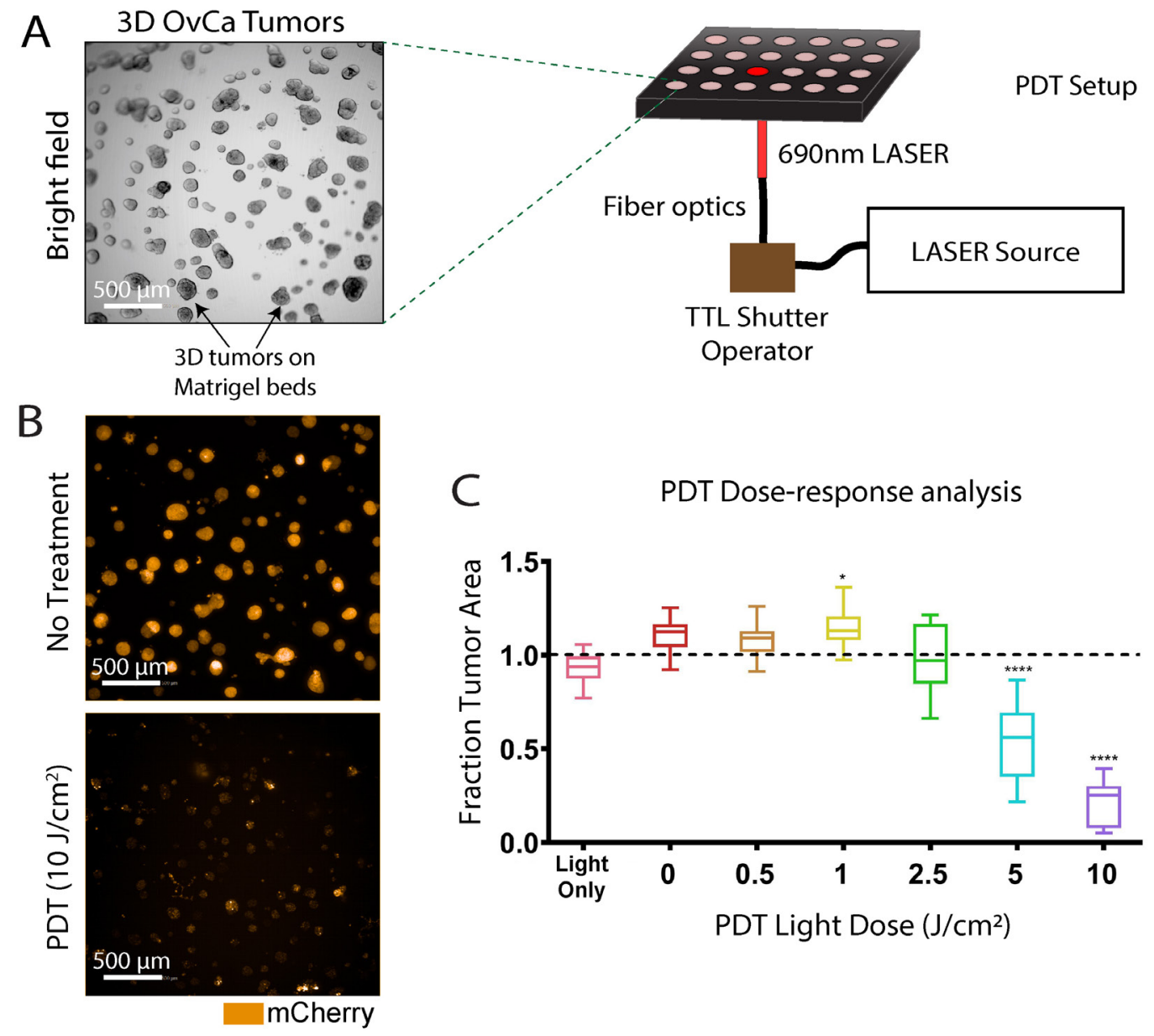

Figure 1. Photodynamic therapy of 3D ovarian cancer nodules. A. A schematic representation of how PDT is performed on the 3D ovarian cancer nodule grown on beds of Matrigel. Scale bar: $500 \mu \mathrm{m}$. B. Representative fluorescence images of 3D tumor nodules following $10 \mathrm{~J} / \mathrm{cm}^{2}$ PDT treatment are shown along with 'no treatment' control. Images were taken 4 days after PDT treatment. Scale bars: $500 \mu \mathrm{m}$. C. Quantification of mCherry-expressing tumor area was performed using a high-content imaging system and presented as 'fraction tumor area' (residual live tumor area) in the graph after normalizing to 'no treatment' control. Images were adapted and reproduced from a published article (Rizvi et al., 2019) with written permission from the publisher.

\section{Notes}

1. Always use LASER safety glasses to protect eyes during PDT irradiation.

2. PDT can be performed outside of a biosafety cabinet while keeping the cellular content sterile in a closed-lid plate.

3. PSs are generally light-sensitive. Thus, PS incubation should be done in the dark. The Multiwell plate can be wrapped in aluminum foil during the incubation process to avoid accidental light exposure. 
4. The treatment response analysis can also be determined if non-fluorescent cells are used. In that case, conventional viability dyes, such as Calcein-AM and propidium iodide can be used as described previously (Anbil et al., 2013).

5. Image analysis can alternatively be done using ImageJ or qVista software (Celli et al., 2014).

6. There are a plethora of PSs and their liposomal and antibody-conjugated formulations. The PS formulation used in a PDT treatment determines the incubation time, light dose, and the wavelength of light irradiation.

7. PDT can also be performed in $2 \mathrm{D}$ monolayer culture which may require optimization of the light dose. In general, the LD50 for 2D culture is lower compared with 3D culture.

8. PDT-treated cells can be harvested for downstream applications (such as qPCR, Flow cytometry, etc.) by depolymerizing Matrigel matrix using Cell Recovery Solution (Corning, 354253) following the manufacturer's protocol.

\section{Recipes}

1. RPMI Growth Medium

a. Add $10 \%(\mathrm{v} / \mathrm{v}) \mathrm{FBS}, 100 \mathrm{IU} / \mathrm{ml}$ Penicillin and $100 \mu \mathrm{g} / \mathrm{ml}$ Streptomycin into $500 \mathrm{ml}$ of RPMI 1640 medium

b. Pre-heat the growth medium at $37^{\circ} \mathrm{C}$ for all the procedures unless otherwise mentioned specifically

2. $4 \%$ Matrigel

Add $400 \mu \mathrm{l}$ Matrigel into $10 \mathrm{ml}$ RPMl growth medium

3. Visudyne working solution ( $1 \mu \mathrm{M}$ verteporfin)

a. First, determine the PS concentration of the Visudyne stock using the following method and then prepare Visudyne working solution by diluting the stock in RPMI growth medium to achieve $1 \mu \mathrm{M}$ PS concentration: Set 'blank' of your UV-Vis instrument with DMSO by scanning from $200 \mathrm{~nm}$ to $800 \mathrm{~nm}$

b. Dilute your Visudyne in an appropriate amount of DMSO that yields an absorbance value at $687 \mathrm{~nm}$ between 0.1 and 1

c. Measure the absorbance of the diluted Visudyne from $200 \mathrm{~nm}-800 \mathrm{~nm}$

d. Calculate your Visudyne concentration using the following equation:

$$
\text { Concentration }=\left(\frac{\left(\text { Absorbance }_{687}-\text { Absorbance }_{800}\right)}{34,895 M^{-1}}\right) \times \text { dilution factor }
$$

\section{Acknowledgments}

This protocol was first reported in a previous publication (Rizvi et al., 2019). We would like to thank the funding sources and the authors of the original publication. The figures were reproduced with 
written permission from the publisher. The authors would like to thank Saad Mohammad and Joseph Swain for helpful discussion.

\section{Competing interests}

The authors declare no competing financial interests.

\section{References}

1. Anbil, S., Rizvi, I., Celli, J. P., Alagic, N., Pogue, B. W. and Hasan, T. (2013). Impact of treatment response metrics on photodynamic therapy planning and outcomes in a three-dimensional model of ovarian cancer. J Biomed Opt 18(9): 098004.

2. Celli, J. P., Rizvi, I., Blanden, A. R., Massodi, I., Glidden, M. D., Pogue, B. W. and Hasan, T. (2014). An imaging-based platform for high-content, quantitative evaluation of therapeutic response in 3D tumour models. Sci Rep 4: 3751.

3. Celli, J. P., Spring, B. Q., Rizvi, I., Evans, C. L., Samkoe, K. S., Verma, S., Pogue, B. W. and Hasan, T. (2010). Imaging and photodynamic therapy: mechanisms, monitoring, and optimization. Chem Rev 110(5): 2795-2838.

4. Goff, B. A., Blake, J., Bamberg, M. P. and Hasan, T. (1996). Treatment of ovarian cancer with photodynamic therapy and immunoconjugates in a murine ovarian cancer model. $\mathrm{Br} J$ Cancer 74(8): 1194-1198.

5. Obaid, G., Broekgaarden, M., Bulin, A. L., Huang, H. C., Kuriakose, J., Liu, J. and Hasan, T. (2016). Photonanomedicine: a convergence of photodynamic therapy and nanotechnology. Nanoscale 8: 12471-12503.

6. Rizvi, I., Bulin, A. L., Briars, E., Anbil, S. and Hasan, T. (2016). Chapter 11: Mind the gap: 3D models in photodynamic therapy. In: Photodynamic Medicine. pp. 197-221.

7. Rizvi, I., Celli, J. P., Evans, C. L., Abu-Yousif, A. O., Muzikansky, A., Pogue, B. W., Finkelstein, D. and Hasan, T. (2010). Synergistic enhancement of carboplatin efficacy with photodynamic therapy in a three-dimensional model for micrometastatic ovarian cancer. Cancer Res 70(22): 9319-9328.

8. Rizvi, I., Nath, S., Obaid, G., Ruhi, M. K., Moore, K., Bano, S., Kessel, D. and Hasan, T. (2019). A Combination of visudyne and a lipid-anchored liposomal formulation of benzoporphyrin derivative enhances photodynamic therapy efficacy in a $3 \mathrm{D}$ model for ovarian cancer. Photochem Photobiol 95(1): 419-429. 